\title{
In-beam spectroscopy of the heaviest elements
}

\author{
Rolf-Dietmar Herzberg ${ }^{a}$ \\ University of Liverpool, UK
}

\begin{abstract}
In-beam spectroscopy provides many powerful tools for the detailed study of nuclear structure. Over the past two decades the coupling of sensitive in-beam spectrometers to recoil separators has allowed the study of weakly populated reaction channels, such as the fusion-evaporation reactions leading to nuclei beyond fermium $(Z=100)$. The methods, observables, and limitations of this approach are discussed.
\end{abstract}

\section{Introduction}

With the recent discovery and naming of elements 113 (nihonium), 115 (moscovium), 117 (tennessine), and 118 (oganesson) [1,2] the seventh row of the chemical table of elements is complete, and the search for the first elements of the 8th row has begun [3-6]. The last element of a row is traditionally a noble gas, which owes its chemical inertness to the strong stabilisation conferred by a completely filled major electron shell. In a nucleus the situation is similar: the nucleus is made up of fermions forming shell structures much like electrons do. On the other hand there are important differences: protons and neutrons form mostly independent shell structures, leading to singly or doubly closed shells. In addition the level density becomes much larger and the separation between shells is not as clear cut as in the atomic case. This leads to the current expectation that the the region of shell-stabilised nuclei owing their relatively long half-lives to large extra binding energies caused by regions of low level density, i.e. the "island of stability", is a complex two-dimensional region on the nuclear landscape whose shores are extremely sensitive to the details of the underlying single particle structure of both protons and neutrons (see, e.g., Ref. [7]).

One of the challenges of nuclear structure physics is therefore to study the single-particle structure of the heaviest nuclei in great detail. The situation is further complicated by the fact that the majority of non-magic nuclei are deformed, which results in a further splitting of the single-particle shells and a dramatic increase in the level density. In 1999 the realisation that the nobelium isotope ${ }^{254}$ No could be synthesized with relatively large production cross sections of $1-2 \mu \mathrm{b}$ led to the first in-beam studies of a nucleus beyond $Z=100[8,9]$, proved that it is indeed a well deformed nucleus and made the whole arsenal of spectroscopic tools available to study nuclei in this region. The state of the art has regularly been reviewed (see references [10-12] and references therein).

In this contribution I will summarise the principle of the experimental techniques, discuss the observables, and look at the inherent limitations of the methods.

\footnotetext{
a e-mail: R.Herzberg@Liverpool.ac.uk
} 


\section{In-beam spectroscopy}

A major experimental challenge is the production of nuclei with more than 100 protons in a fusion reaction. The dominant background through fission and quasi-fission takes an ever larger fraction of the total reaction cross section, with the fusion-evaporation channels of interest becoming (much) less than $10^{-6}$ of the total. Tagging techniques developed in the 1990s [13] allowing a selection of the fusion products from this overwhelming background are crucial. They work by placing an array of detectors around the target position to record the radiation emitted promptly from the reaction. The reaction products are then passed into a recoil separator where they are selected over the unwanted reaction products and unreacted primary beam. Products of interest are detected in the separator focal plane and a time correlation with the promptly emitted radiation at the target position can be carried out using the well known flight time through the separator. For an illustration of the operating principle see, e.g., references [10-12].

The prompt detector array has mostly consisted of Ge-detectors for $\gamma$-ray spectroscopy. The initial experiment used GAMmasPHERE at Argonne National Laboratory [8, 14] and a number of similar detector arrays have since been used, notably the JUROGAM I and II arrays at the accelerator laboratory of the University of Jyväskylä.

This general detection scheme can be easily expanded. A conversion electron detector can be used either with or without $\gamma$-ray detection capability. An example of the latter is the SACRED spectrometer [15, 16], an example of the former is the SAGE spectrometer [17], where a highly segmented Si pixel detector for electrons is combined with the JUROGAM II array of Ge-detectors.

A further breakthrough came in 2002 when a new calorimetric approach [18] allowed selection of isomeric states populated in the initial reaction. Provided that the isomeric halflives were at least as long as the flight time through the separator, this opened the door to the study of isomers in one of the most fertile isomer hunting grounds in the nuclear chart [19-21].

To illustrate the scope and reach of in-beam spectroscopic methods in the heaviest nuclei Fig. 1 summarises the available data in elements from curium upwards. The figure has been updated from $[11,12]$ and modified to indicate those nuclei studied using in-beam spectroscopic methods to date.

\subsection{Observables}

In-beam spectroscopy has been the workhorse of nuclear spectroscopy for many years, and a large number of spectroscopic techniques are available (see, e.g., Ref. [22]). The most important is the ability to use $\gamma$-ray coincidence techniques to build up even complex level schemes, which are indispensable for the reliable assignment of configurations, spins, and parities. From the energy spacings in the ground-state rotational band one can deduce the moment of inertia as a function of the rotational frequency of the nucleus. The nucleons sitting in the single-particle orbitals with the highest angular momentum experience the largest Coriolis force and will be the first ones to break their paired state and align their spins with the rotational axis. This "backbending" behaviour is directly observable in the moment of inertia and can be compared to model calculations probing the location and occupation of the high- $j$ single-particle orbitals and the pairing strength of the nucleus. This is especially important as the main difference between microscopic-macroscopic models and fully self-consistent models is the location of the high- $j$ orbitals in relation to the other singleparticle configurations [23, 24]. Figure 2 shows the dynamic moments of inertia for even-even isotopes with $N=146-154$. 


\section{Proton Number}

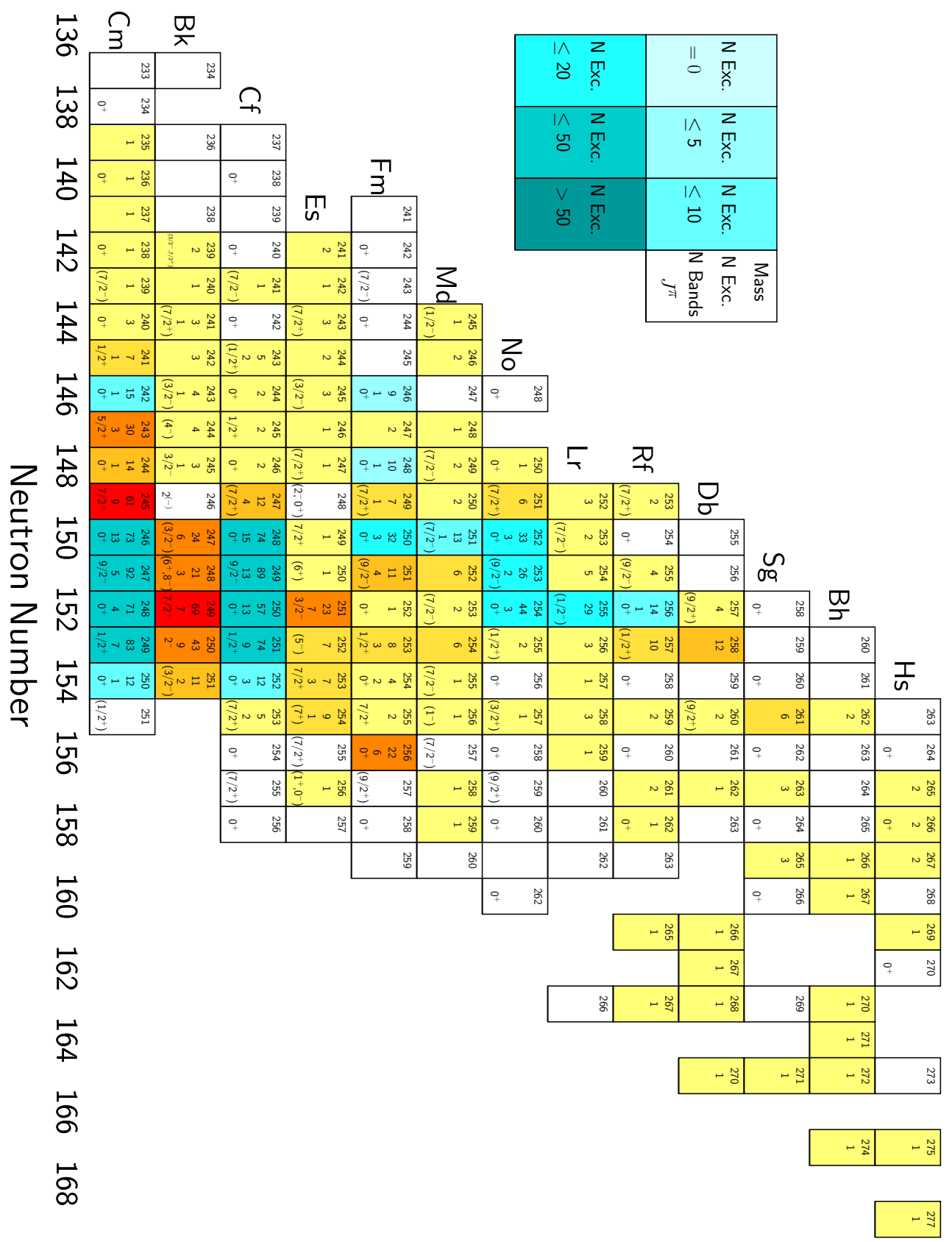

Figure 1. Schematic summary of evaluated nuclear data available in the ENSDF data base nuclei from $\mathrm{Cm}$ to $\mathrm{Hs}$. We give for each isotope the mass number, the number of known excited levels, the number of assigned rotational bands, and the ground state spin. The blue boxes indicate those nuclei where in-beam spectroscopy has been carried out, the darker shades correspond to more levels identified. (Figure based on Fig. 1 in [11].) 


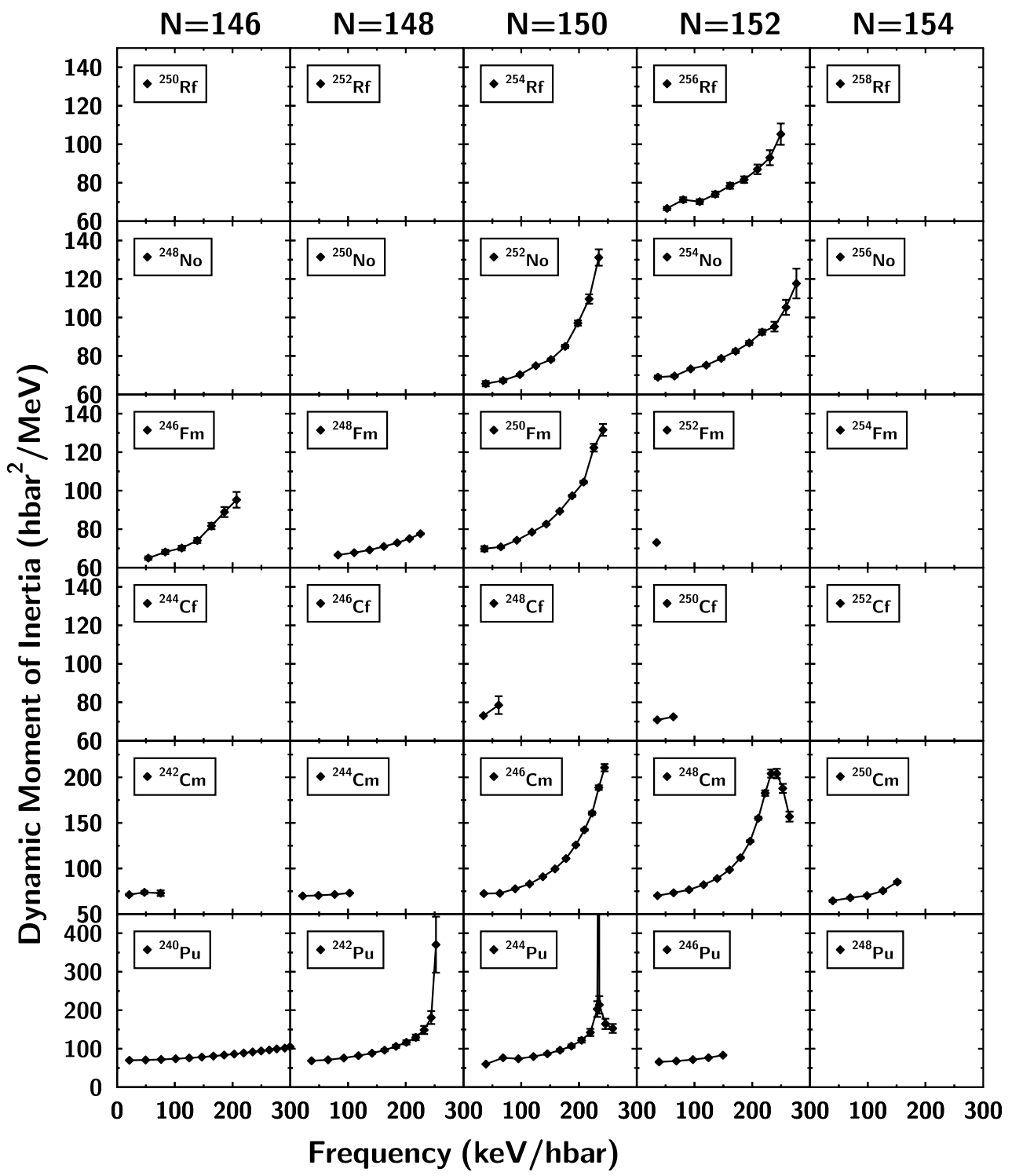

Figure 2. Dynamic moments of inertia for nuclei around ${ }^{254}$ No plotted against angular frequency. The different upbending behaviours are clearly seen. (Adopted from Fig. 18 in [11], data for ${ }^{256} \mathrm{Rf}$ is taken from [25], data for ${ }^{252} \mathrm{Fm}$ is taken from [26].)

If one looks at the ground state rotational bands of ${ }^{250} \mathrm{Fm},{ }^{252,254} \mathrm{No}$, and ${ }^{256} \mathrm{Rf}$ one can observe the difference in upbending behaviour between the $N=150$ isotones and the $N=152$ isotones [25]. In these nuclei the $i_{13 / 2}$ proton orbitals and the $j_{15 / 2}$ neutron orbitals are partially filled and are the first proton and neutron orbitals expected to align, respectively. Any difference in behaviour is therefore very sensitive to the relative position of these orbitals in relation to the Fermi surface.

In odd-mass isotopes the rotational band built on the ground state carries even more information. In general one will observe a strongly coupled system of two bands, one for each signature, connected with intraband $E 2$ transitions and interband mixed $E 2 / M 1$ 
$[514] 7 / 2^{-}$

$47 / 2^{-}$

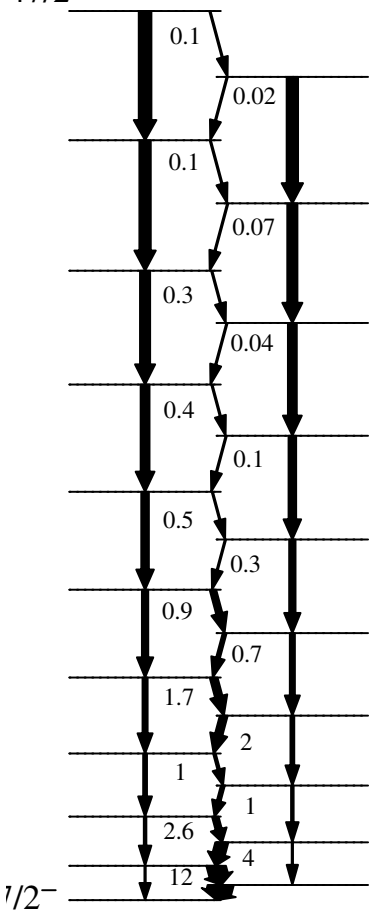

$[633] 7 / 2^{+}$

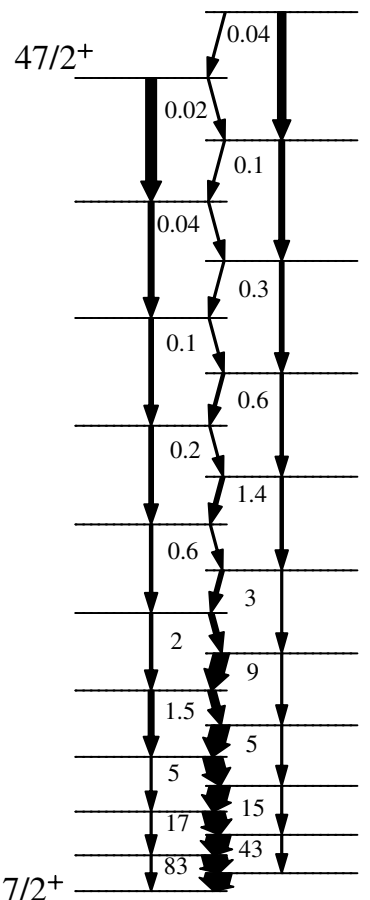

$[521] 1 / 2^{-}$

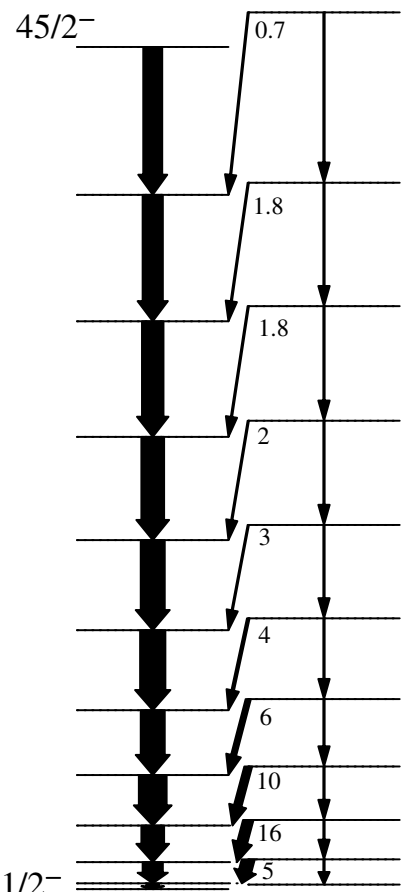

Figure 3. Schematic decay patterns for the three configuration candidates for rotational bands in ${ }^{251} \mathrm{Md}$. The numbers labelling the states indicate the ratio $T(M 1) / T(E 2)$. (Taken from [27].)

transitions. The branching ratio between the interband/intraband transitions is sensitive to both the quadrupole moment of the band and the gyromagnetic factors of the groundstate configuration. Thus a measurement of the branching ratios in such bands can provide strong evidence to assign a specific configuration to the ground state, or, more generally, the band head. Such examples have been ${ }^{251} \mathrm{Md}$ [27] and ${ }^{253}$ No [28-30]. Figure 3 illustrates the expected branching ratios in the case of ${ }^{251} \mathrm{Md}$ [27]. Here model calculations for the three configurations most likely to form the ground state, namely [514]7/2 $2^{-},[633] 7 / 2^{+}$, and $[521] 1 / 2^{-}$give very different and distinctive intensity patterns. On the basis of this pattern the ground-state configuration was assigned as built on the $[521] 1 / 2^{-}$single proton orbital [27].

This technique can equally well be applied to bands built on high- $K$ isomeric states in even-even nuclei, such as the band built on the $K^{\pi}=8^{-}$isomers in ${ }^{252} \mathrm{No}$ [31] and ${ }^{250} \mathrm{Fm}$ $[32,33]$, resulting in an assignment of the two-quasineutron configuration $8^{-}\left([624] 7 / 2^{+}{ }_{v} \otimes\right.$ $[734] 9 / 2^{-} v$ ) for the isomers. In ${ }^{254}$ No the configuration of the $3^{+}$excited state populated in the decay of the lowest $8^{-}$isomer was identified as $3^{+}\left([521] 1 / 2^{-} \otimes[514] 7 / 2^{-}{ }_{\pi}\right)$. This is significant as the $[521] 1 / 2^{-} \pi$ proton orbital stems from the spherical $f_{5 / 2}$ singleparticle level located above the spherical $Z=114$ gap [19]. Although one starts to run into problems with statistics, it is possible to assign configurations in this way. Indeed, new methods to deal with extremely low statistics data have been developed for just this purpose [34]. 


\subsection{Limitations}

In-beam spectroscopy typically uses a recoil separator to pull the weak prompt signal from the dominant background by using a coincidence with the recoiling nucleus. However, the maximum beam current usable in these experiments is set by the maximum event rate observed at the target position. This is entirely dominated by unwanted background from fission and transfer channels, largely independent of the desired reaction. Thus the main limitation at cross sections below the nanobarn level comes from the total number of recoils produced on a target with a beam current limited by the prompt event rate. At present the lowest cross section for meaningful in-beam $\gamma$-ray spectroscopy in an even-even transfermium nucleus is $17 \mathrm{nb}$, reached in the reaction of ${ }^{50} \mathrm{Ti}$ on ${ }^{208} \mathrm{~Pb}$ targets [25].

Modern $\gamma$-ray tracking arrays such as AGATA [35] or GRETA/GRETINA [36, 37] can push that limit down by at least one order of magnitude. They do this by tracking every $\gamma$-ray interaction in a full germanium shell and then reconstructing the number and energy of all simultaneously incoming $\gamma$ rays using tracking algorithms. This is possible because the Compton scattering process gives a well defined relationship between the scattering angle and the deposited energy in each Compton scattering event. Thus these tracking arrays can make use of pile-up events which have to be discarded in non-tracking spectrometers. This allows the detectors to be operated at a much higher event rate, and thus allow a large beam intensity on the target.

\section{References}

[1] P.J. Karol, R.C. Barber, B.M. Sherrill, E. Vardaci, T. Yamazaki, Pure Appl. Chem. 88, 139 (2016).

[2] P.J. Karol, R.C. Barber, B.M. Sherrill, E. Vardaci, T. Yamazaki, Pure Appl. Chem. 88, 155 (2016).

[3] Ch. E. Düllmann et al., GSI Scientific Report 2011, GSI Report 2012-1, p. 206.

[4] S. Hofmann et al., GSI Scientific Report 2008, GSI Report 2009-1, p. 131.

[5] S. Hofmann et al., GSI Report 2012-1, p. 205.

[6] Yu.Ts. Oganessian et al., Phys. Rev. C 79, 024603 (2009).

[7] M. Bender et al., Phys. Lett. B515 42 (2001).

[8] P. Reiter et al., Phys. Rev. Lett. 82, 509 (1999).

[9] M. Leino et al., Eur. Phys. J. A 6, 63 (1999).

[10] R.-D. Herzberg, J. Phys. G30, R123 (2004).

[11] R.-D. Herzberg and P.T. Greenlees, Prog. Part. Nucl. Phys. 61, 674 (2008).

[12] Ch. Theisen et al., Nucl. Phys. A944, 333 (2015).

[13] E.S. Paul et al., Phys. Rev. C 51, 78 (1995).

[14] I.-Y. Lee, Nucl. Phys. A520, 641c (1990).

[15] P.A. Butler et al., Nucl. Instrum. Meth. A381, 433 (1996).

[16] H. Kankaanpää et al., Nucl. Instrum. Meth. A534, 503 (2004).

[17] J. Pakarinen et al., Eur. Phys. J. A 50, 53 (2014).

[18] G.D. Jones, Nucl. Instrum. Meth. A488, 471 (2002).

[19] R.-D. Herzberg et al., Nature 442, 896 (2006).

[20] R.-D. Herzberg and D.M. Cox, Radiochim. Acta 99, 441 (2011).

[21] G.D. Dracoulis et al., Rep. Prog. Phys. 79, 076301 (2016).

[22] H. Morinaga, In-Beam Gamma-Ray Spectroscopy (Elsevier Science Ltd, 1977).

[23] M. Bender et al., Nucl. Phys. A723, 354 (2003).

[24] A.V. Afanasjev et al., Phys. Rev. C 67, 024309 (2003).

[25] P.T. Greenlees et al., Phys. Rev. Lett. 109, 012501 (2012). 
[26] M. Asai, priv. comm. (2016).

[27] A. Chatillon, et al., Phys. Rev. Lett. 98, 132503 (2007).

[28] P. Reiter et al., Phys. Rev. Lett. 95, 032501 (2005).

[29] R.-D. Herzberg et al., Eur. Phys. J. A 42, 333 (2009).

[30] A. Mistry et al., to be published.

[31] B. Sulignano et al., Phys. Rev. C 86, 044318 (2012).

[32] J.E. Bastin et al., Phys. Rev. C 73, 024308 (2006).

[33] P.T. Greenlees et al., Phys. Rev. C 78, 021303(R) (2008).

[34] E. Parr et al., Eur. Phys. J. A 48, 134 (2012).

[35] S. Akkoyun et al., Nucl. Instrum. Meth. A668, 26 (2012).

[36] I.Y. Lee et al., Rep. Prog. Phys. 66, 1095 (2003).

[37] I.Y. Lee, Nucl. Phys. A746, 255c (2004). 